\title{
Session 1: Advances in Bioenergy Feedstocks and Plant Science
}

\author{
C. Neal Stewart Jr. • Timothy Tschaplinski
}

Published online: 28 March 2009

(C) Humana Press 2009

While the stars of biofuel biotechnology are engineered conversion enzymes or microbes, at the root of all biofuels are biomass feedstocks. Whether ethanol, longer-chain alcohols, or biodiesel, the feedstocks for these platforms will mainly be higher plants well into the foreseeable future. Even though arguments surface for developing algal feedstocks, it remains to be seen whether infrastructure and capital costs for ponds and processing are prohibitively expensive for algae to become a feasible reality in the mid-to-long-range future.

Researchers who made presentations in the Advances in Bioenergy Feedstocks and Plant Science session also understand the importance of emerging higher-plant feedstocks, such as switchgrass (Panicum virgatum), as evidenced by four of the six papers that focused on this species. Considering that switchgrass can deliver approximately 600 gallons per acre [1] using today's conversion technologies, approximately twice that of current corn-grainto-ethanol platforms, and that there are a plethora of biotechnology tools and developing germplasm resources, this focus is justified [2].

\section{The Presentations}

\section{Switchgrass}

Burton English discussed "Developing a dedicated energy crop for Tennessee" and was peppered with numerous questions from the audience. There was good reason for this interest. Dr. English and colleagues have been performing agronomic field trials of several varieties of switchgrass for over 4 years in Tennessee. His project, initiated in 2003, has examined practical agronomic issues such as the effect of variety, land topography, nutrient input, and seeding rates on biomass production. They have shown that yields of over 10 dry tons per acre of unmodified Alamo are reasonable expectations for western Tennessee.

C. N. Stewart Jr. $(\bowtie)$

Oak Ridge National Laboratory, Oak Ridge, TN, USA

e-mail: nstewart@uncg.edu

T. Tschaplinski

University of Tennessee, Knoxville, TN, USA 
These data are important for setting baselines for future switchgrass feedstock development using biotechnology and breeding.

A presentation made by a team of researchers from the USDA-ARS and the University of Nebraska overviewed "Switchgrass, lignin, enzymes and ethanol." With extensive switchgrass experience, they have selected populations that vary in in vitro dry matter digestibility. Acid-digested lignin content was correlated with ethanol yield. Of special interest in their analysis were plants for low lignin. They showed altered biosynthesis enzyme activities and $\mathrm{S}$ and $\mathrm{G}$ lignin amounts. These traits were genotype-dependent. However, since the complete lignin biosynthetic pathway for switchgrass is incompletely understood, we cannot completely decipher endogenous variation yet, but these switchgrass populations and data represent tremendous resources for future feedstock development.

Towards the goal of better understanding switchgrass lignin biosynthesis, Joshua Yuan and colleagues presented "Genomic, transcriptomic, proteomic and functional analysis of candidate genes for bioenergy feedstock improvement." To better understand lignin biosythesis in switchgrass, researchers are using the extensive genomics resources in Arabidopsis thaliana and rice for comparative genomic approaches. For example, extensive transcriptomic approaches in multiple plant species showed how lignin biosynthesis and other cell-wall-associated gene expression changes in various organs and developmental stages. Coupled with proteomics approaches, there are vast opportunities to identify potential gene targets for improving feedstocks using systems biology.

Noble Foundation researcher Fang Chen and colleagues reported on the "Development of low-lignin biomass feedstock for improved biofuel production." Building upon their long-term experience in altering alfalfa (Medicago sativa) for lower lignin for improved forage, this group is now also focusing on genetically improved feedstocks for biofuels. As with the presentations above, a main target is switchgrass. In their transgenic alfalfa work, the expression of several lignin biosynthesis genes was manipulated. The most important finding was that there was a direct negative relationship between lignin content and saccharification potential. Therefore, it is clear that as the genomics of switchgrass lignin biosynthesis is better elucidated, it will enable the engineering of feedstocks for lower lignin content that should result in more fuel per acre from advanced feedstocks.

\section{Altering Photosynthesis}

The presentation "Maximizing photosynthetic yield by increasing sink strength" by Christer Jannson of LBNL cross-cut among plant species and feedstocks. The research goal was to change a fundamental property of plants - that is, to increase carbon assimilation to increase biomass production. The authors thrust is to better understand regulatory networks and molecular switches that control photosynthate partitioning. As above, this group strives to use the power of systems biology to understand a basic aspect of plant biology that should translate to feedstock development. One gene family that has received attention is SUSIBA transcription factors. Manipulating one or more of these transcription factors may result in a change in downstream gene expression that alters source-sink carbon relationships. Doing so has the potential to greatly enhance the ability to more precisely control plant growth.

\section{A Biodiesel Crop}

Finally, the only industry contribution to the session was "Engineering the nation's first dedicated biofuel crop." Don Panter of Sustainable Oils envisions the annual mustard Camelina sativa to be a superior biodiesel feedstock that also holds potential as a source of 
biochemicals and bioproducts as well. This company, a partner of Targeted Growth, is using conventional breeding with marker-assisted selection and genetic engineering to improve both oil and agronomic qualities of Camelina, which is not currently used for food, and thus avoids the food-fuel dilemma.

Prospects

Clearly the Feedstocks session gave us a glimpse into the future, in which yields and the variety of products coming from new feedstocks are great. Indeed, new biotechnologies and systems biology tools should pave the way for dramatic changes in the future of biomass feedstocks. We expect to see rapid progress with switchgrass and other cellulosic feedstocks, and also a diversification of biodiesel feedstocks. The latter will likely be more of a niche industry in the US since the demand for biodiesel is less, but related fuels that are critical, such as jet fuel, could drive future research to meet these needs in a sustainable way.

\section{References}

1. Shubert, C. (2006). Nature Biotechnology, 24, 777-784.

2. Yuan, J. S., et al. (2008). Trends in Plant Science, 13, 421-429. 